\title{
Bacteriophages as an alternative to the use of antibiotics in poultry breeding
}

\author{
Teresa Guerrero $^{\mathrm{a}}$, Sonia Zapata ${ }^{\mathrm{b}}$ \\ a Universidad San Francisco de Quito, Instituto de Microbiología, Quito, Ecuador \\ e-mail: etguerrero@asig.com.ec \\ ${ }^{\mathrm{b}}$ Universidad San Francisco de Quito, Instituto de Microbiología, Quito, Ecuador \\ e-mail: szapata@usfq.edu.ec
}

\begin{abstract}
Bacterial resistance to antibiotics is an emerging problem worldwide; antimicrobials used in animal husbandry are the most important factor in the propagation of resistance genes, because the amount of antibiotics used in the animal industry is greater than in the medical field. Since phages constitute a safe alternative for the control of human pathogenic bacteria in food, this project aimed to isolate and characterize Salmonella spp. bacteriophages and use them to reduce the presence of this pathogen in poultry at the farm level, without the use of antibiotics. We obtained phage cocktails that showed lytic activity in vitro against $83 \%$ of the Salmonella strains isolated from poultry farms. The cocktails with the greatest spectrum of activity against Salmonella were obtained from nearby polluted rivers. We were able to eliminate the pathogen from the chicken intestine when phages were applied 48 hours before the animals were slaughtered, but it is necessary to maintain a permanent monitoring of the phage's lytic activity against resistant strains of Salmonella.
\end{abstract}

\section{Introduction}

Bacterial resistance to antibiotics is an emerging problem worldwide (World Health Organization, 2015). The use of antimicrobials is responsible for resistance in commensal bacteria of animals that have never received antibiotics, due to horizontally transferred genes (Ventola, 2015). The antimicrobials used as growth promoters or prophylactic agents in animal husbandry are the most important factor in the propagation of resistance genes, since the amount of antibiotics used in the animal industry is greater than in the medical field (Davies \& Davies, 2010). The use of phages constitutes a safe alternative for the control of human pathogenic bacteria, with the consequent reduction in the incidence of foodborne diseases, and the costs of rejecting food products when there is an outbreak.

Phages are used as a biological alternative to eliminate pathogenic bacteria from food, because they do not affect eukaryotic cells and are safe for beneficial microorganisms that are also found in food and in the human intestinal tract. They provide a high degree of safety due to their specificity of host bacteria 
since they produce rapid host death (Fiorentin, Vieira, \& Barioni, 2005; Huang et al., 2018; Pang, Lambertini, Buchanan, Schaffner, \& Pradhan, 2017). These viruses are widely distributed in human and animal feces, sewage and the environment of edible animals (Dueñas el al., 2017).

Salmonella is an important pathogen, mainly transmitted to humans through contaminated food, and causing a high rate of morbidity and mortality worldwide. The zoonotic Salmonella serotypes that cause foodborne diseases are found in the intestinal tract of warm-blooded animals, such as birds, which allows their passage to the meat during the slaughter of the animal and the processing, considering chicken meat as the main infectious source of non-typhoidal Salmonella.

The aim of this project was to isolate and characterize bacteriophages of Salmonella spp. and use them to reduce the presence of this pathogen in poultry at the farm level, without the use of antibiotics.

\section{Materials and Methods}

Phage cocktails were isolated from river waters, sewage from chicken processing facilities and samples from chicken beds. The enrichment was carried out following the methodology of Benson (1980).

The host range and the titer of the phage cocktails were determined following the methodology of Huang et al. (2018) and those that were specific only against Salmonella spp. were applied in a poultry farm where pilot trials are carried out. The application routes were orally (with water) and by sprinkling.

\section{Results and Discussion}

We obtained phage cocktails that showed lytic activity in vitro against $83 \%$ of Salmonella strains isolated from poultry farms (serotypes Infantis, Braenderup and Saintpaul).

The phage cocktails with the highest spectrum of activity against our Salmonella strains were obtained from contaminated rivers. There were positive results (elimination of Salmonella in the chicken intestine) when phages were applied 48 hours before the animals were slaughtered.

The results were similar when phages were administered orally and when combined with spray, because the evaluation was performed only on the cecum of slaughtered chickens.

\section{Conclusions}

From sewage near poultry farms, we isolated phage cocktails which eliminated Salmonella when they were administered to poultry. No difference was found when applying phages orally and when combined with spraying. In future trials we will take samples of chicken coops to determine if the spray is effective to eliminate the pathogen from the poultry litters.

As bacteria have mechanisms by which they can become immune to virus attack, such as the CRISPRCas system (Shariat et al., 2015), it is necessary to maintain a permanent monitoring of lytic activity of the phages against resistant Salmonella strains.

Currently, we are beginning the isolation and characterization of individual phages from the best cocktails.

\section{References}

Benson, H. (1980) Microbial applications, a laboratory manual in general microbiology, 3rd ed. Iowa: Wm. C. Brown Company Publishers.

Davies, J., \& Davies, D. (2010). Origins and Evolution of Antibiotic Resistance. Microbiology and 
Molecular Biology Reviews, 74(3), 417-433. https://doi.org/10.1128/MMBR.00016-10

Dueñas, F., Rivera, D., Toledo, V., Tardone, R., Hervé-Claude, L. P., Hamilton-West, C., \& Switt, A. I. M. (2017). Short communication: Characterization of Salmonella phages from dairy calves on farms with history of diarrhea. Journal of Dairy Science, 100(3), 2196-2200. https://doi.org/10.3168/jds.201611569

Fiorentin, L., Vieira, N. D., \& Barioni, W. (2005). Oral treatment with bacteriophages reduces the concentration of Salmonella Enteritidis PT4 in caecal contents of broilers. Avian Pathology, 34(3), 258263. https://doi.org/10.1080/01445340500112157

Huang, C., Virk, S. M., Shi, J., Zhou, Y., Willias, S. P., Morsy, M. K., ... Li, J. (2018). Isolation, characterization, and application of Bacteriophage LPSE1 against Salmonella enterica in Ready to Eat (RTE) Foods. Frontiers in Microbiology, 9(MAY), 1-11. https://doi.org/10.3389/fmicb.2018.01046

Pang, H., Lambertini, E., Buchanan, R. L., Schaffner, D. W., \& Pradhan, A. K. (2017). Quantitative Microbial Risk Assessment for Escherichia coli O157:H7 in Fresh-Cut Lettuce. Journal of Food Protection, 80(2), 302-311. https://doi.org/10.4315/0362-028X.JFP-16-246

Ventola, C. L. (2015). The antibiotic resistance crisis: part 1: causes and threats. P \& T: A PeerReviewed Journal for Formulary Management (2015), 40(4), 277-283. https://doi.org/Article

World Health Organization. (2015). Global Antimicrobial Resistance Surveillance System. Retrieved from http://apps.who.int/iris/bitstream/10665/188783/1/9789241549400_eng.pdf?ua=1 